\title{
Avaliação de Desempenho do TCP em Sistemas VBLAST Codificados
}

\author{
Alessandro P. de Oliveira, Marcelo E. Pellenz, Richard D. Souza e Manoel C. Penna
}

\begin{abstract}
Resumo-As próximas gerações de sistemas de comunicação sem fio certamente irão incorporar as técnicas MIMO de maneira a propiciar serviços multimídia com altas taxas de transmissão. O protocolo de controle de transmissão (TCP) é geralmente implementado na camada de transporte para garantir comunicação confiável dos dados. Por esta razão, a otimização do desempenho do protocolo TCP para sistemas MIMO é atualmente uma importante área de pesquisa. Neste trabalho investigamos diversos esquemas VBLAST codificados, com diferentes eficiências espectrais, de maneira a melhorar o desempenho fim-a-fim do protocolo TCP para aplicações cliente-servidor e peer-to-peer. Avaliamos o desempenho de sete esquemas codificados usando códigos convolucionais com diferentes taxas de codificação e ordem de memória. A melhor estratégia de transmissão para cada valor de relação sinal-ruído é determinada.
\end{abstract}

Palavras-Chave-MIMO, VBLAST, TCP, RLP, Desempenho, Redes Sem Fio.

Abstract-The next generation of wireless communications systems will likely incorporate MIMO techniques in order to provide higher data rate multimedia services. Moreover, the transmission control protocol (TCP) is generally employed at the transport layer for reliable data communication. Thus, the optimization of TCP performance over MIMO systems is currently an important research area. In this paper, we investigate several VBLAST coded schemes, with different spectral efficiencies, in order to improve the end-to-end TCP performance for client-server and peer-to-peer applications. We evaluate seven coded schemes using convolutional codes with different coding rates and memories. The best transmission strategy for each value of signal-to-noise ratio is determined.

Keywords-MIMO, VBLAST, TCP, RLP, Performance, Wireless Networks.

\section{INTRODUÇÃO}

Os novos padrões para redes sem fio, como por exemplo os padrões IEEE 802.16 (WiMAX) [1] e IEEE802.11n (WiFi) [2], incorporam a tecnologia MIMO (Multiple-Input Multiple-Output) de maneira a aumentar a eficiência espectral e/ou a confiabilidade do enlace. O desempenho dos sistemas MIMO pode ser consideravelmente maior que os sistemas SISO (Single-Input Single-Output) tradicionais [3], [4]. Os sistemas MIMO podem ser implementados de duas maneiras distintas. A primeira abordagem visa aumentar a eficiência espectral ou a capacidade do sistema utilizando multiplexação espacial, fornecendo altas taxas de transmissão. Nesta estratégia, denominada de BLAST (Bell-Labs Layered Space-Time) [4], [5], as diferentes antenas são utilizadas para

Alessandro P. de Oliveira, Marcelo E. Pellenz e Manoel C. Penna , PPGIa, Pontifícia Universidade Católica do Paraná (PUCPR), Curitiba-PR, Brasil, E-mails: \{alessandro, marcelo, penna\}@ppgia.pucpr.br. Richard D. Souza, CPGEI, Universidade Tecnológica Federal do Paraná (UTFPR), CuritibaPR, Brasil, E-mail: richard@cpgei.cefetpr.br. Este trabalho foi parcialmente financiado pelo CNPq (472977/2007-5). transmitir diferentes símbolos de informação codificados, ao mesmo tempo. A segunda abordagem melhora a confiabilidade do enlace, explorando o ganho de diversidade espacial. Estes sistemas geralmente implementam códigos de bloco espaçotemporais, denominados STBC (Space Time Block Codes) [6], [7]. Nesta abordagem as diferentes antenas transmitem diferentes símbolos codificados do mesmo símbolo de informação, num dado instante de tempo. A comunicação se torna mais confiável, mas a taxa de transmissão pode ser penalizada, sendo ainda menor que em sistemas SISO, dependendo da taxa de codificação do STBC.

Os novos padrões WiMAX e WiFi são tecnologias alternativas para o acesso em banda larga a Internet usando redes sem fio em malha (Wireless Mesh Networks - WMNs). Em WMNs, de maneira similar as redes Ad-Hoc, cada nó pode operar não apenas como um terminal cliente, mas também como um roteador. Portanto, isso requer que garantias de qualidade de serviço (Quality of Service - QoS) sejam oferecidas aos consumidores, possibilitando aplicações multimídia como Voz sobre IP (VoIP), Vídeo sob Demanda (VoD) e classes diferenciadas de serviço. Isso tem motivado o projeto crosslayer de sistemas, onde as camadas superiores de protocolo trocam informações com a camada física e a camada de controle de acesso ao meio (MAC) para melhor explorar os recursos da rede. Existem diversos trabalhos recentemente publicados na área de projeto crosslayer, que investigam o impacto da camada física nas camadas superiores de protocolo, em especial as interações entre a camada física e o protocolo TCP da camada de transporte [8].

Em redes cabeadas o desempenho do TCP é afetado pelo congestionamento da rede, que causa descarte de pacotes nos roteadores devido ao estouro de filas de roteamento. O protocolo TCP detecta o processo de descarte de pacotes e reduz a taxa de transmissão. Em redes sem fio, existe uma causa adicional para a perda de pacotes, que são os erros de transmissão na camada de enlace de rádio. Contudo, o protocolo TCP não é capaz de distinguir entre as duas causas de perdas que podem ocorrer.

Além disso, em redes sem fio o enlace de rádio apresenta tipicamente altas taxas de erro de quadro (Frame Error Rate - FER), afetando severamente o protocolo TCP [8], [9]. Para melhorar o desempenho do TCP em enlaces sem fio, um mecanismo ARQ (Automatic Repeat-reQuest) de retransmissão é geralmente implementado na camada de enlace. Os segmentos de dados do TCP são fragmentados pelo protocolo de enlace de rádio (Radio Link Protocol - RLP) e os fragmentos corrompidos são retransmitidos na camada RLP antes de serem entregues à camada superior. Contudo, este método pode introduzir atrasos e variações na taxa de transmissão se um 
número excessivo de retransmissões ocorrer. Isso vai induzir perdas de pacotes em surtos, reduzindo a taxa útil do enlace [9]. Portanto, uma solução baseada apenas em ARQ não é suficiente para melhorar o desempenho do TCP em enlaces sem fio.

Alguns trabalhos recentemente publicados investigam o desempenho do TCP em sistemas de transmissão MIMO. Em [10] foi proposta uma técnica de otimização para o sistema MIMO, onde um grupo de antenas é selecionado para receber maior alocação de potência que os outros grupos, com o objetivo de maximizar o desempenho do TCP. Em [11] foi investigado o desempenho do TCP em sistemas VBLAST não codificados e esquemas STBC. No estudo realizado em [11] foi considerado apenas um único esquema de modulação. Foram analisados os impactos do ARQ e das técnicas de combinação de pacotes em ambos os sistemas. Os resultados obtidos em [11] demonstram que para baixos valores de relação sinal-ruído (SNR), o sistema STBC é superior ao VBLAST e para valores altos de SNR, o VBLAST supera o desempenho do STBC.

Neste trabalho investigamos diferentes esquemas VBLAST codificados para diferentes tipos de modulação e propomos uma estratégia de transmissão adaptativa para ser usada em redes sem fio com múltiplos saltos e redes em malha. Esta estratégia melhora o desempenho fim-a-fim do TCP para aplicações cliente-servidor e peer-to-peer. Os esquemas codificados utilizam códigos convolucionais com diferentes taxas e ordens de memórias. O objetivo é avaliar qual sistema desempenha melhor em uma faixa de relação sinal-ruído (SNR) específica. Como resultado derivamos um conjunto de regras para selecionar o melhor esquema VBLAST codificado em função da relação sinal-ruído média entre os nós que estão se comunicando.

Este artigo está organizado da seguinte forma: A Seção II apresenta uma breve revisão do protocolo TCP. Na Seção III discutimos os problemas de desempenho do TCP em enlaces sem fio. A arquitetura do sistema VBLAST é descrita na Seção IV. Na Seção V apresentamos o esquema de transmissão proposto e avaliamos o desempenho do TCP/RLP para diferentes estratégias de codificação. As conclusões são apresentadas na Seção VI.

\section{Protocolo TCP}

O protocolo TCP [13] é um protocolo de transporte orientado a conexão. O TCP é responsável por adaptar a taxa de transmissão às condições de banda disponíveis no enlace de comunicação, evitando o congestionamento da rede e criando uma conexão confiável através da retransmissão de pacotes perdidos. A confiabilidade é obtida através do envio de segmentos de confirmação (Acknowledgment - ACK) transmitidos pelo receptor em resposta aos segmentos de dados já recebidos. Os ACKs indicam ao transmissor a numeração do próximo segmento esperado no receptor. Um pacote é considerado perdido quando três ACKs repetidos para o mesmo pacote chegam à fonte, ou quando um ACK não é recebido na fonte dentro de um período de tempo específico (retransmissão por timeout). Quando um segmento chega fora de ordem ao receptor, são gerados ACKs duplicados e isso não é considerado uma perda. Um segmento é retransmitido tipicamente após a ocorrência de três ACKs duplicados, mesmo que o tempo para retransmissão por timeout não tenha expirado no transmissor.

De maneira a se controlar a taxa de transmissão e evitar o congestionamento da rede, o número de pacotes que podem ser transmitidos sem o recebimento do ACK é limitado por um parâmetro denominado janela de congestionamento (Congestion Window - CW). O tamanho da CW é controlado dinamicamente de acordo com o estado da rede [14]. Para uma nova conexão, ou quando uma retransmissão é gerada por timeout, a CW é ajustada para a fase de inicialização lenta (slow start). A cada vez que um ACK é recebido, a CW é incrementada de um segmento. Durante a fase slow start a CW aumenta até que um determinado valor limite é atingido ou até que ocorra uma retransmissão por timeout. Quando isso acontece, a fase slow start termina e uma segunda fase denominada congestion avoidance inicia. Nesta fase a CW tem um crescimento linear. Um mecanismo adicional denominado fast retransmit e fast recovery também é implementado. Neste caso, um segmento perdido é detetado e recuperado antes que ocorra a retransmissão por timeout. Portanto a taxa de transmissão não é reduzida tanto quanto ocorreria quando a retransmissão é gerada por timeout, porque existe uma deteção e retransmissão antecipada do pacote perdido, aumentando o desempenho do TCP.

Existem diversas variantes do protocolo TCP. Na versão Tahoe, sempre que uma perda é detectada, o tamanho da janela de congestionamento reduz para um e a fase slow start inicia novamente. Em outras versões bastante utilizadas como o TCP Reno e New Reno, o tamanho da CW se reduz para um apenas se uma perda for detetada por timeout. Quando uma perda é detetada pela ocorrência de ACKs repetidos, o tamanho da $\mathrm{CW}$ se reduz pela metade. Assim a fase de slow start não é iniciada novamente e o protocolo se mantêm na fase congestion avoidance.

\section{Desempenho do TCP EM Enlaces Sem Fio}

O protocolo TCP foi originalmente projetado para operação em redes cabeadas. O backbone da rede Internet é composto por enlaces de fibra ótica de alta velocidade que conectam os roteadores da rede. Neste nível, a transmissão dos dados pode ser considerada como livre de erros. As perdas de pacote são usualmente geradas por estouro de memória nas filas de roteamento. Uma parte menos confiável da rede é a rede de acesso. A rede de acesso cabeada pode ser implementada usando-se fibra ótica, cabo coaxial ou par trançado. Nestes cenários os mecanismos de controle do TCP descritos na Seção II são considerados bastante efetivos para garantir bom desempenho em termos de taxa de transmissão, mesmo sob congestionamentos moderados da rede. O canal definido pelo par trançado não pode ser assumido como livre de erros e apresenta na prática uma taxa de erro de bit considerável induzida pelo ruído, interferência e distorção. Contudo, este canal ainda é bastante previsível se comparado com canais sem fio.

Em contraste, a comunicação sem fio é afetada por problemas adicionais, como o desvanecimento, o que pode resultar 
em altas taxas de erro de bit. O desempenho do TCP em redes sem fio pode ser severamente reduzido, pois perdas adicionais de pacotes são induzidas pelo canal de rádio. O TCP interpreta estas perdas como sendo causadas por congestionamento na rede. Neste caso o TCP executa os mecanismos de controle de congestionamento, reduzindo o taxa de transmissão útil (throughput) da conexão e aumentando o atraso fim-a-fim para transferência dos dados.

Em alguns casos, a redução do throughput é desnecessária porque o canal de rádio apresenta desvanecimentos intensos, que geram altas taxas de erro de bit e muitas perdas de pacotes, mas que duram pouco tempo. Nesta situação todos os pacotes são perdidos e o protocolo TCP reduz o número de pacotes injetados na rede. Basicamente existem três estratégias para minimizar a redução do throughput do TCP em enlaces de rádio [11]. A primeira estratégia é utilizar um mecanismo de retransmissão (ARQ) na camada de enlace de rádio de maneira a evitar que o TCP reinicie na fase de slow start. Retransmissões na camada de enlace são possíveis porque o tempo de timeout do TCP é um valor suficientemente grande para permitir a retransmissão de alguns quadros perdidos. Este procedimento é implementado pelo protocolo de enlace de rádio (RLP). Muitos padrões de comunicação sem fio de nova geração, como por exemplo o UMTS [12], implementam o RLP.

O RLP realiza fragmentação e remontagem de pacotes na camada de enlace e utiliza filas de armazenagem no transmissor e receptor. Existe uma temporização para timeout para cada quadro perdido no receptor. Este temporizador é usualmente configurado para o tempo de transmissão de quatro quadros RLP. Quando ocorre o timeout para um determinado quadro, ou um quadro RLP não consecutivo é recebido, uma perda é identificada. Neste caso, o receptor envia um NACK (Negative Acknowledgment) para o transmissor, indicando que um quadro está faltando, que então procede a retransmissão. Este processo é executado para um mesmo quadro até um máximo de $n$ ocorrências de timeout (usualmente entre 3 e 10 vezes) ou até que o quadro correto seja recebido. Após as $n$ tentativas, se o quadro RLP ainda estiver faltando, a camada de enlace de rádio descarta todos os quadros precedentes, referentes ao mesmo segmento TCP. Assim, o protocolo da camada de transporte (TCP) inicia o seu próprio processo de recuperação.

\section{Arquitetura do Sistema VBLAST}

Considere um sistema MIMO com $n_{T}$ antenas transmissoras e $n_{R}$ antenas receptoras, onde $n_{R} \geq n_{T}$. Na arquitetura VBLAST [5], o fluxo de dados é dividido em $n_{T}$ sub-fluxos, usando um conversor serial-paralelo, onde cada sub-fluxo é modulado e codificado separadamente. Os sub-fluxos são então transmitidos simultaneamente pelas $n_{T}$ antenas transmissoras. Cada antena receptora recebe todos os sinais transmitidos das $n_{T}$ antenas transmissoras. Então os sinais são processados para detectar cada sub-fluxo transmitido. Seguindo a notação usada em [11], o sinal recebido no sistema VBLAST pode ser expresso como

$$
\mathbf{y}=\mathbf{H} \cdot \mathbf{s}+\mathbf{n}
$$

onde $\mathbf{y}=\left[y_{1}, y_{2}, \ldots, y_{n_{R}}\right]^{T}$ e $\mathbf{s}=\left[s_{1}, s_{2}, \ldots, s_{n_{T}}\right]^{T}$ são vetores coluna representando os símbolos recebidos e transmitidos, respectivamente. Os símbolos transmitidos, $s_{i}$, são pontos normalizados (energia unitária) de uma constelação de sinais. No vetor de ruído recebido, $\mathbf{n}=\left[n_{1}, n_{2}, \ldots, n_{n_{R}}\right]^{T}$, as componentes $n_{i}$ são variáveis aleatórias Gaussianas com média zero e variância $\sigma^{2}=n_{T} /(2 \cdot \rho)$, onde $\rho$ é a relação sinal-ruído. O canal é representado por uma matriz $\mathbf{H}$ de dimensão $n_{R} \times n_{T}$, onde os elementos $h_{i j}$ representam o ganho complexo do canal entre a $j$-ésima antena transmissora e a $i$ ésima antena receptora. Para a deteção do sinal assumimos que apenas o receptor conhece a matriz $\mathbf{H}$ do canal.

$\mathrm{O}$ detetor VBLAST ótimo é o detetor de máxima verossimilhança, cuja complexidade computacional cresce exponencialmente com o número de antenas transmissoras. $\mathrm{Na}$ prática, dois detetores de baixa complexidade são comumente implementados no receptor: Zero-Forcing (ZF) e Minimum Mean Square Error (MMSE). O algoritmo ZF possui uma implementação mais simples que o MMSE, a um custo de um menor desempenho. Ambos os detetores são assintoticamente similares em altas relações sinal-ruído [15].

Neste trabalho utilizamos o detetor ZF, onde o símbolo inicialmente detectado é subtraído do sinal recebido para gerar um cancelamento sucessivo de interferência. Este procedimento é repetido até que todos os símbolos transmitidos sejam detetados. As etapas do algoritmo deteção ZF são as seguintes [5]:

a) $\overline{\mathbf{H}}=\mathbf{H}$;

b) Calcular a matriz pseudoinversa $\mathbf{G}=\overline{\mathbf{H}}^{+}$;

c) Determinar o vetor de cancelamento para o $i$-ésimo subfluxo: $\mathbf{w}=\mathbf{G}(i,:)$;

d) Detetar o símbolo corrente: $\mathbf{r}=\mathbf{w} \cdot \mathbf{y}$;

e) Quantizar: $\hat{s}=Q(\mathbf{r})$;

f) Subtrair o símbolo estimado do sinal recebido: $\mathbf{y}=\mathbf{y}-$ $\overline{\mathbf{H}}\left(:, k_{i}\right) \cdot \hat{s}$;

g) Zerar os elementos da $i$-ésima coluna de $\mathbf{H}$;

h) Repetir o processo do passo b) até que todos os símbolos tenham sido detectados.

\section{ESQUema Proposto}

O objetivo deste trabalho é investigar e propor um esquema de transmissão adaptativo da camada física, que maximiza o throughput do TCP dependendo da faixa SNR de operação. Tal abordagem nos permite introduzir uma degradação de desempenho suave na faixa de operação do VBLAST. É importante ressaltar que esta estratégia é diferente do que simplesmente alternar entre esquemas VBLAST e STBC conforme proposto em [11], onde foi utilizada uma modulação fixa. Consideramos esquemas VBLAST usando diferentes taxas de codificação e diferentes ordens de modulação. A arquitetura proposta para o sistema VBLAST é mostrada na Figura 1. Assumimos um esquema MIMO com $n_{T}=n_{R}=4$ antenas. Os quatro subfluxos de dados são codificados separadamente usando um código convolucional. Os parâmetros dos sistemas VBLAST simulados são apresentados na Tabela I.

A taxa de erro de quadro (Frame Error Rate - FER) para estes esquemas foi obtida através de simulação usando o software MATLAB. Para avaliar o throughput do TCP, os valores 

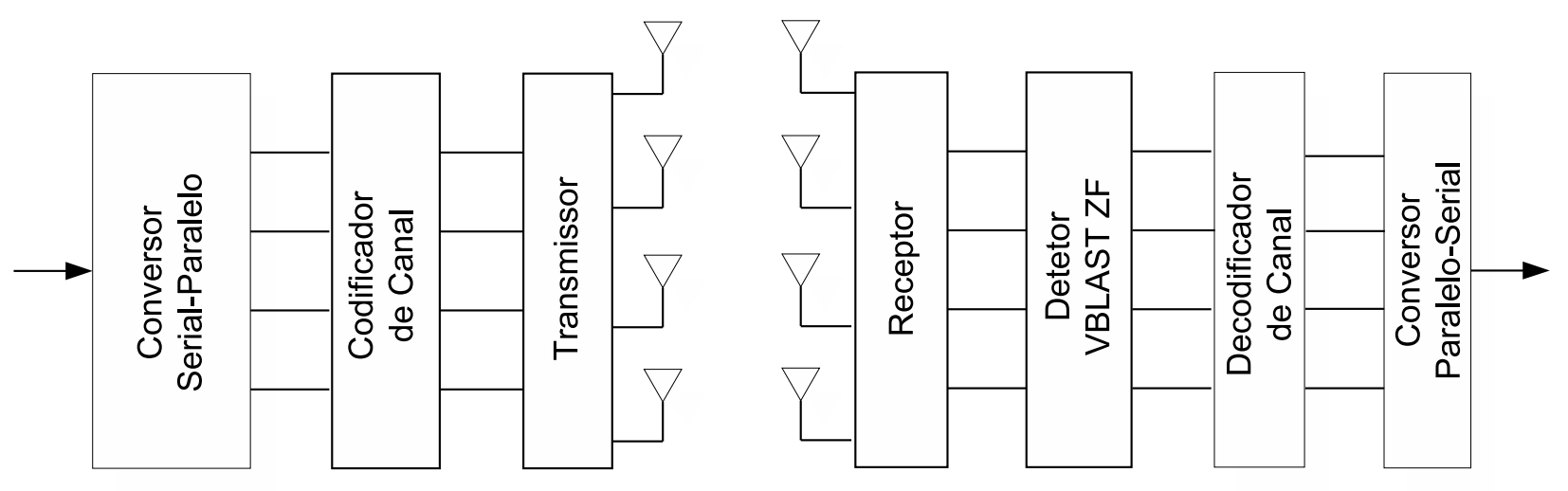

Fig. 1. Arquitetura do Sistema Proposto.

\begin{tabular}{||c|c|c|c|c|}
\hline \hline Esquema & Modulação & Taxa do Código $r$ & Memória $m$ & bits/s/Hz \\
\hline S1 & 8-QAM & $2 / 3$ & 3 & 8 \\
\hline S2 & 8-QAM & $1 / 2$ & 2 & 6 \\
\hline S3 & 8-QAM & $1 / 3$ & 2 & 4 \\
\hline S4 & QPSK & $2 / 3$ & 3 & $16 / 3$ \\
\hline S5 & QPSK & $1 / 3$ & 2 & $8 / 3$ \\
\hline S6 & BPSK & $1 / 2$ & 2 & 2 \\
\hline S7 & BPSK & $1 / 3$ & 2 & $4 / 3$ \\
\hline \hline
\end{tabular}

TABELA I

ESQUEMAS CODIFICADOS VBLAST IMPLEMENTAdos.

de FER obtidos da simulação da camada física foram usados para modelar o enlace sem fio no cenário de rede apresentado na Figura 2. O cenário é constituído por seis, onde os nós 2 e 3 se comunicam através do enlace sem fio. Os demais enlaces são cabeados. Um arquivo de dados é transmitido usando o protocolo de aplicação FTP (File Transfer Protocol) através do TCP. A pilha de protocolos TCP/IP/LL/PHY, implementado para os nós 0 e 5, é responsável pela execução do FTP e a transferência dos dados do arquivo. A pilha de protocolos PPP/RLP/LL/PHY, implementado para os nós 2 e 3, transmite os dados através do enlace sem fio. O padrão RLP implementado na camada de enlace de rádio é o protocolo 3GPP2 RLP3 [16]. A versão TCP implementada foi o protocolo TCP Reno. O tamanho máximo do segmento de dados (Maximum Segment Size - MSS) usado foi de 536 bytes. Este é o tamanho padrão do MSS usado na camada RLP [16]. O cenário de rede foi simulado no software NS2 [17].

\begin{tabular}{||c|c|c|c||}
\hline \hline Esquema & bits/s/Hz & $\begin{array}{c}\text { Taxa do Enlace } \\
\text { (kbps) }\end{array}$ & $\begin{array}{c}\text { Quadro RLP } \\
\text { (bytes) }\end{array}$ \\
\hline S1 & 8 & 64 & 160 \\
\hline S2 & 6 & 48 & 120 \\
\hline S3 & 4 & 32 & 80 \\
\hline S4 & $16 / 3$ & $128 / 3$ & 106 \\
\hline S5 & $8 / 3$ & $64 / 3$ & 53 \\
\hline S6 & 2 & 16 & 40 \\
\hline S7 & $4 / 3$ & $32 / 3$ & 26 \\
\hline
\end{tabular}

TABELA II

Parâmetros de Simulação da Camada de Enlace.

Para compararmos corretamente os esquemas simula-

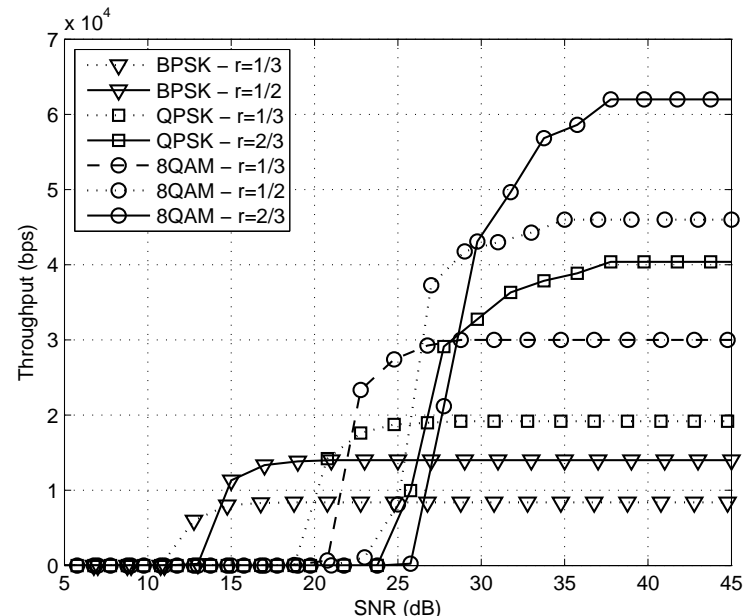

Fig. 3. Desempenho do TCP para os esquemas VBLAST da Tabela I.

\begin{tabular}{||c|c||}
\hline \hline Esquema Selecionado & Faixa de SNR \\
\hline BPSK 1/3 & $S N R \leq 14.8 d B$ \\
\hline BPSK 1/2 & $14.8 d B \leq S N R \leq 21 d B$ \\
\hline QPSK 1/3 & $21 d B \leq S N R \leq 22.7 d B$ \\
\hline 8QAM 1/3 & $22.7 d B \leq S N R \leq 26.7 d B$ \\
\hline 8QAM 1/2 & $26.7 d B \leq S N R \leq 29.6 d B$ \\
\hline 8QAM 2/3 & $S N R \geq 29.6 d B$ \\
\hline
\end{tabular}

TABELA III

FAIXAS DE OPERAÇÃO PARA OS ESQUEMAS CODIFICADOS.

dos, que possuem diferentes eficiências espectrais, uma compensação na taxa de transmissão do enlace sem fio deve ser feita na simulação. Para o esquema com maior eficiência espectral (8QAM; $r=2 / 3$ ), a capacidade do enlace foi especificada como sendo de $64 \mathrm{kbps}$, conforme adotado em [11]. Os outros esquemas possuem uma alocação de capacidade de enlace proporcional, conforme mostrado na Tabela II.

Na Figura 3 apresentamos os resultados de simulação para o throughput do TCP em função da SNR para os diferentes esquemas codificados VBLAST. Destas curvas podemos identificar a estratégia de codificação apropriada que deve ser implementada para cada faixa de SNR. Os esquemas selecionados são apresentados na Tabela III. É importante 


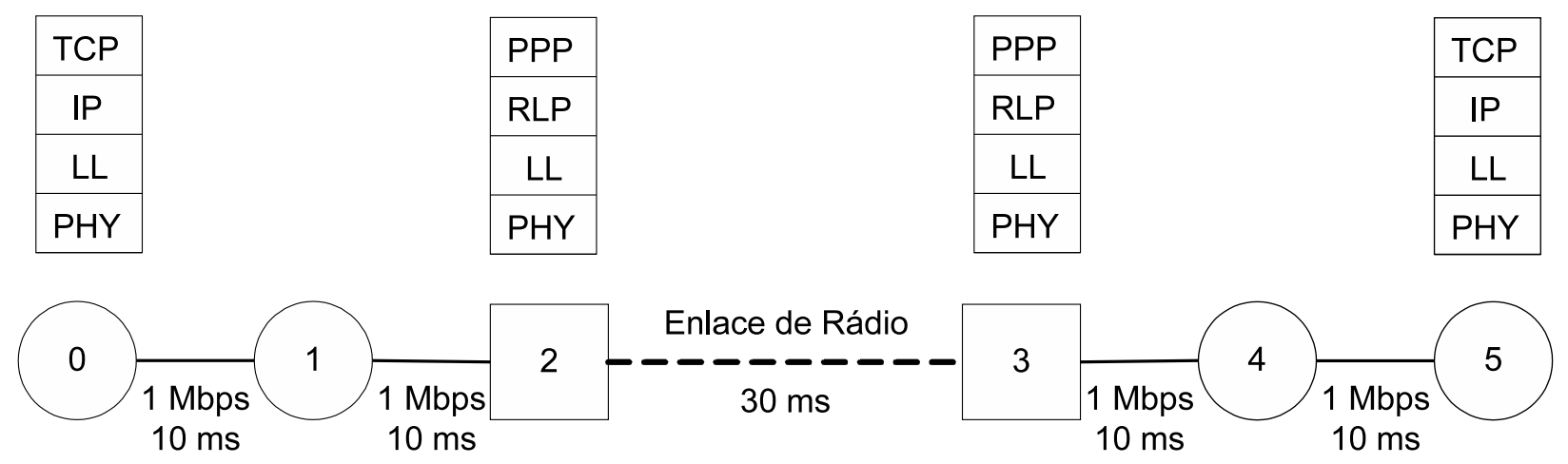

Fig. 2. Cenário de Rede Implementado no Simulador NS2.

ressaltar que o esquema (S4) usando QPSK e taxa $r=2 / 3$ não foi selecionado para nenhuma faixa de operação. Neste trabalho implementamos o detetor ZF para o VBLAST. A utilização do detetor MMSE pode melhorar significativamente o desempenho de todos os esquemas, deslocando as curvas de throughput para a esquerda na Figura 3. Porém, as conclusões em relação aos resultados não se modificam.

Analisando a Figura 3 podemos observar que haveria uma perda significativa de desempenho se o método de seleção não fosse utilizado. Considere por exemplo que o sistema opere apenas com o esquema 3 da Tabela I (8QAM; $r=1 / 3$ ). Para a faixa de SNR abaixo de $20 \mathrm{~dB}$ o desempenho do TCP é próximo a zero. Para a faixa de SNR acima de 28 $\mathrm{dB}$ o throughput do TCP atinge uma taxa de saturação de $30 \mathrm{kbps}$. Fica claro que um desempenho muito melhor é obtido quando o esquema apropriado de modulação e codificação é selecionado para cada faixa de SNR, conforme indicado na Tabela III.

Na Figura 4 é apresentada a curva de operação para o throughput do protocolo TCP operando com os esquemas propostos para cada faixa de SNR. A linha vertical pontilhada delimita a faixa ótima de operação de cada esquema apresentado na Tabela I. É importante ressaltar, baseado nos resultados de [11], que na faixa de operação onde o esquema VBLAST apresenta throughput superior ao STBC, o esquema otimizado proposto neste trabalho permite melhor ainda mais o throughput do VBLAST.

\section{CONCLUSÕES}

Neste trabalho investigamos o desempenho das camadas TCP/RLP para transmissão usando diferentes esquemas VBLAST codificados. Os esquemas utilizam códigos convolucionais com diferentes taxas e ordens de modulação. O resultado principal derivado das simulações apresentadas na Figura 3 é a possibilidade de modificação da taxa de codificação e da ordem da modulação de maneira a se maximizar o throughput do TCP, dependendo da faixa de operação de SNR. Esta abordagem permite introduzir uma degradação suave de desempenho na faixa de operação do VBLAST. É importante ressaltar que a estratégia de seleção proposta pode propiciar melhoras significativas de desempenho para as camadas TCP/RLP do que simplesmente um chaveamento entre esquemas fixos VBLAST e STBC. Alguns aspectos

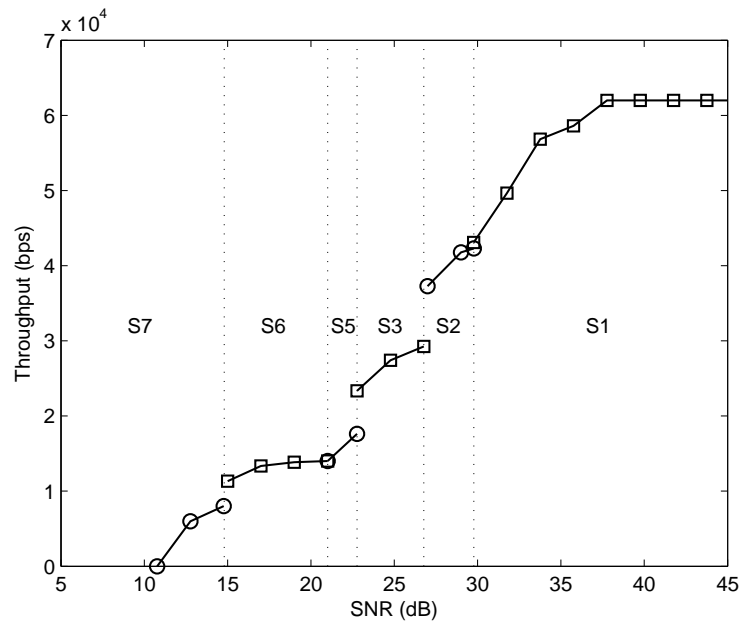

Fig. 4. Desempenho do TCP usando as estratégias otimizadas de transmissão.

ainda devem ser investigados em trabalhos futuros, como outros esquemas de codificação de canal e a proposta de um protocolo específico para adaptar dinamicamente a estratégia de transmissão de acordo com as condições do canal.

\section{REFERÊNCIAS}

[1] IEEE Standard 802.16-2004, "IEEE Standard for Local and metropolitan area networks Part 16: Air Interface for Fixed Broadband Wireless Access Systems," May 2004.

[2] IEEE Draft 802.11n, "IEEE Approved Draft Standard for Information Technology-Telecommunications - Part 11: Wireless LAN Medium Access Control (MAC) and Physical Layer (PHY) specifications," Sept. 2007.

[3] G. J. Foschini, "Layered Space-Time Architecture for Wireless Communication in a Fading Environment when Using Multi-Element Antennas", Bell Labs Technical Journal, vol. 1, no. 2, pp. 41-59, 1996.

[4] G. J. Foschini and M. J. Gans, "On Limits of Wireless Communications in a Fading Environment when Using Multiple Antennas,"Wireless Personal Communications, vol. 6, no. 3, pp. 311-335, March 1998.

[5] P. W. Wolniansky, G. J. Foschini, G. D. Golden and R. A. Valenzuela, "V-BLAST: An Architecture for Realizing Very High Data Rates over the Rich-Scattering Wireless Channel," Proc. Int. Symp. on Signals, Systems, and Electronics, pp. 295-300, Sept. 1998.

[6] S. M. Alamouti, "A Simple Transmit Diversity Technique for Wireless Communications," IEEE Journal of Selected Areas in Communications, vol. 16 , no. 8, pp. 1451-1458, Oct. 1998.

[7] V. Tarokh, H. Jafarkhami and A. R. Calderbank, "Space-Time Block Codes from Orthogonal Designs," IEEE Trans. on Information Theory, vol. 45, no. 5, pp. 1456-1467, July 1999. 
[8] H. Balakrishnan, V. N. Padmanabham, S. Seshan and R. H. Katz, "A Comparison of Mechanisms for Improving TCP Performance over Wireless Links," Proc. of ACM SIGCOMM, August 1996.

[9] M. C. Chan and R. Ramjee, "TCP/IP Performance over 3G Wireless Links with Rate and Delay Variation," Proc. of The ACM Annual International Conference on Mobile Computing and Networking (Mobicom), Sept. 2002.

[10] A. Milani, V. Tralli and M. Zorzi, "On the Use of Per-Antenna Rate and Power Adaptation in V-BLAST Systems for Protocol Performance Improvement," Proc. of IEEE Vehicular Technology Conf. (VTC), vol. 4, pp. 2126-2130, Set. 2002.

[11] A. L. Toledo and X. Wang, "TCP Performance over Wireless MIMO Channels with ARQ and Packet Combining,'IEEE Transactions on Mobile Computing, vol. 5, no. 3, pp. 208-223, March 2006.

[12] Third Generation Partnership Project, RLC Protocol Specification (3G TS 25.322), 1999.

[13] W. Stevens, "RFC2001: TCP Slow Start, Congestion Avoidance, Fast Retransmit and Fast Recovery Algorithm,'Internet Eng. Task Force Request for Comments, Jan. 1997.

[14] M. Allman, S. Floyd and C. Partridge. "RFC3390: Increasing TCP's initial window", Internet Eng. Task Force (IETF) - Request for Comments, August 2002.

[15] D. Shiu, J. M. Kahn, "Scalable Layered Space-Time Codes for Wireless Communications: Performance Analysis and Design Criteria,"Proc. of The IEEE Wireless Communications and Networking Conference (WCNC), pp. 159-163, 1999.

[16] Third Generation Partnership Project 2 (3GPP2), "Data Service Options for Spread Spectrum Systems: Radio Link Protocol Type 3,'June 2004.

[17] Network Simulator 2, http://www.isi.edu/nsnam/ns, 2007. 